Article

\title{
A New Consequence of Van Aubel's Theorem
}

\section{Dasari Naga Vijay Krishna}

Department of Mathematics, Narayana Educational Instutions, Machilipatnam, Bengalore, India; vijay9290009015@gmail.com

Abstract: In this short paper we study some properties of the lines associated with van Aubel's theorem in the special case when squares are replaced with equilateral triangles constructed on the sides of an arbitrary quadrilateral.

Keywords: van Aubel's theorem; iso Ortho Diagonal Quadrilateral; equilateral triangle

\section{INTRODUCTION}

\section{Van Aubel's Theorem::}

Consider an arbitrary quadrilateralABCD, the quadrilateral $\mathrm{S}_{1} \mathrm{~S}_{2} \mathrm{~S}_{3} \mathrm{~S}_{4}$ formed by joining the four corresponding centers $\mathrm{S}_{1}, \mathrm{~S}_{2}, \mathrm{~S}_{3}, \mathrm{~S}_{4}$ of the squares thus constructed on each side of $\mathrm{ABCD}$ is an Iso Ortho Diagonal quadrilateral ( see Figure.1)[5]. That is $S_{1} S_{3}=S_{2} S_{4}$ and $S_{1} S_{3} \perp S_{2} S_{4}$.

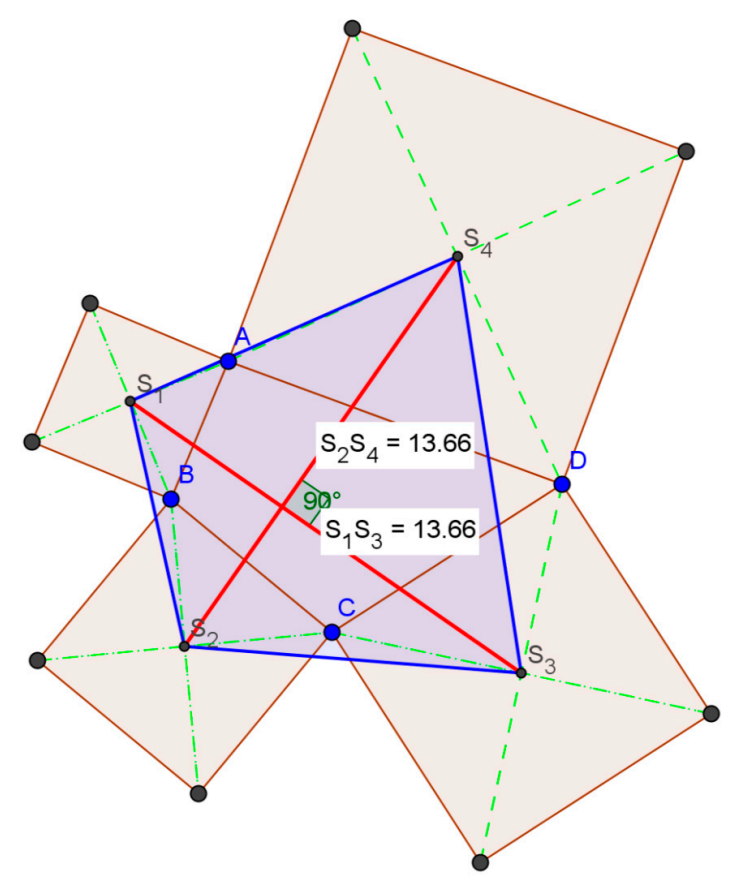

Figure.1

From Figure.1, it is clear $S_{1} S_{3}=S_{2} S_{4}$ and $S_{1} S_{3} \perp S_{2} S_{4}$ 
The theorem we just stated above is attributed to Van Aubel (Von Aubel in [Gardner, p. 176-178]) could also be found in [de Villiers, Yaglom, Finney] among others.

In this article, we study the properties of the lines $S_{1} S_{3}$ and $S_{2} S_{4}$ when the squares are replaced with equilateral triangles, our present study about the special case of Van Abel's theorem when the squares are replaced with equilateral triangles and further generalization is not actually new, since some of the authors studied about this earlier in 90's ( can be found in [3], [4], [6], [7], [8] and [9] ). Even though it is not a new study and the results presented in this article seems to be very elementary but are quite new and interesting.

In this article we present two results related to the lines $S_{1} S_{3}$ and $S_{2} S_{4}$ when the squares are replaced with equilateral triangles ( $\triangle \mathrm{ABP}, \triangle \mathrm{BCQ}, \triangle \mathrm{CDR}$ and $\triangle \mathrm{DAS}$ ) such as "The pair of lines $\left(S_{1} S_{3}, Q S\right)$ and $\left(S_{2} S_{4}, P Q\right)$ are perpendicular" and "The four points common to the lines $S_{1} S_{3}, S_{2} S_{4}, P Q$ and $R S$ are concyclic". Clearly the second result is the consequence of first .

\section{Preliminaries}

We use the following lemma's in proving the above mentioned results.

\section{Lemma-1}

If $\triangle A B C$ is an equilateral triangle with center $S$ and $M$ be any point in the plane of the triangle then $3 S M^{2}=A M^{2}+B M^{2}+C M^{2}-A B^{2}$

\section{Proof:}

It is very obvious to prove above lemma if we use the relation present in [1].

\section{Lemma-2}

A convex quadrilateral $A B C D$ is orthodiagonal if and only if $A B^{2}+C D^{2}=B C^{2}+A D^{2}$

\section{Proof:}

The proof of above lemma is present in [2].

\section{Lemma-3}

If Equilateral triangles $\triangle A B P, \triangle B C Q, \triangle C D R$ and $\triangle D A S$ are constructed entirely lies out wards on the sides $A B=a, B C=b, C D=c$ and $A D=d$ of an arbitrary convex quadrilateral $A B C D$ whose area is $\triangle$ then

$$
A Q^{2}+P Q^{2}+C S^{2}+R S^{2}=D Q^{2}+R Q^{2}+B S^{2}+P S^{2}=2\left(a^{2}+b^{2}+c^{2}+d^{2}-\Delta\right)
$$

\section{Proof:}


In order to prove above lemma, we proceed as follows by considering a quadrilateralABCD mentioned in figure. 2

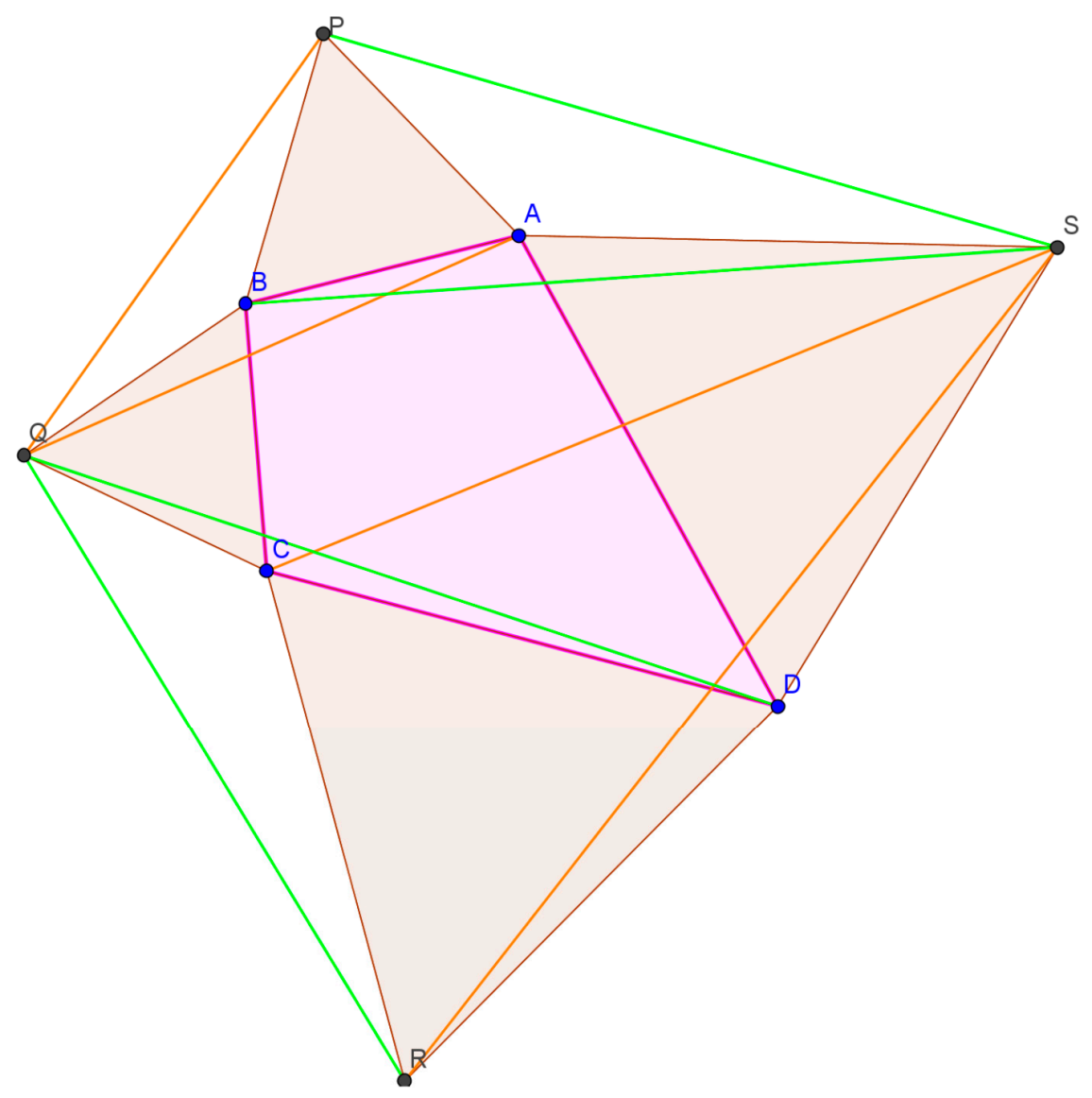

Figure.2

By applying the cosine rule for the triangles $\triangle \mathrm{ABQ}, \triangle \mathrm{PBQ}, \triangle \mathrm{SDC}$, and $\Delta \mathrm{SDR}$ we get

$$
\begin{aligned}
& A Q^{2}=A B^{2}+B Q^{2}-2 A B \cdot B Q \cdot \cos (\angle \mathrm{ABQ})=a^{2}+b^{2}-2 a b \cos (60+B) \\
& P Q^{2}=B P^{2}+B Q^{2}-2 B P \cdot B Q \cdot \cos (\angle \mathrm{PBQ})=a^{2}+b^{2}-2 a b \cos (240-B) \\
& C S^{2}=C D^{2}+D S^{2}-2 C D \cdot D S \cdot \cos (\angle \mathrm{CDS})=c^{2}+d^{2}-2 c d \cos (60+D) \\
& R S^{2}=R D^{2}+D S^{2}-2 R D \cdot D S \cdot \cos (\angle \mathrm{RDS})=c^{2}+d^{2}-2 c d \cos (120+D)
\end{aligned}
$$

By computing $(1)+(2)+(3)+(4)$, we get

$A Q^{2}+P Q^{2}+C S^{2}+R S^{2}$ 


$$
=2\left[a^{2}+b^{2}+c^{2}+d^{2}-a b(\cos (60+B)+\cos (240-\mathrm{B})-c d(\cos (60+D)+\cos (120+\mathrm{D})]\right.
$$

With the help of transformations of the angles and also using the fact $2 \Delta=a b \sin B+c d \sin D$,

(5) can be further simplified so as to prove $A Q^{2}+P Q^{2}+C S^{2}+R S^{2}=2\left(a^{2}+b^{2}+c^{2}+d^{2}-\Delta\right)$

In the similar manner we can prove $D Q^{2}+R Q^{2}+B S^{2}+P S^{2}==2\left(a^{2}+b^{2}+c^{2}+d^{2}-\Delta\right)$

Hence $A Q^{2}+P Q^{2}+C S^{2}+R S^{2}=D Q^{2}+R Q^{2}+B S^{2}+P S^{2}$

We now state and prove our main results.

\section{Main Results}

\section{Theorem-1}

If $S_{1}, S_{2}, S_{3}$ and $S_{4}$ are the centers of the equilateral triangles $\triangle A B P, \triangle B C Q, \triangle C D R$ and $\triangle D A S$ are constructed entirely lies out wards on the sides $A B=a, B C=b, C D=c$ and $A D=d$ of an arbitrary convex quadrilateral $A B C D$ then the lines $P R, Q S$ are respectively perpendicular to the lines $S_{2} S_{4}, S_{1} S_{3}$. That is $S_{1} S_{3} \perp Q S, S_{2} S_{4} \perp P R$

\section{Proof:}

To prove the line $\mathbf{S}_{\mathbf{1}} \mathbf{S}_{\mathbf{3}}$ is perpendicular to the line $\mathrm{QS}$, it is enough to prove that the quadrilateral $\mathrm{S}_{1} \mathrm{QS}{ }_{3} \mathrm{R}$ is a orthodiagonal,

To prove this using lemma-2 it is enough to prove $S_{1} Q^{2}+S_{3} S^{2}=S_{1} S^{2}+S_{3} Q^{2}$

Using lemma-1, we have $3 S M^{2}=A M^{2}+B M^{2}+C M^{2}-A B^{2}$

Fix $\mathrm{S}$ as $\mathrm{S}_{1}, \mathrm{~S}_{3}$ and $\mathrm{M}$ as $\mathrm{Q}, \mathrm{S}$ we get

$$
\begin{aligned}
& 3 S_{1} Q^{2}=A Q^{2}+B Q^{2}+P Q^{2}-a^{2} \\
& 3 S_{1} S^{2}=A S^{2}+B S^{2}+P S^{2}-a^{2} \\
& 3 S_{3} Q^{2}=C Q^{2}+D Q^{2}+R Q^{2}-c^{2} \\
& 3 S_{3} S^{2}=C S^{2}+D S^{2}+R S^{2}-c^{2}
\end{aligned}
$$

So as to prove (6), it is enough to prove $A Q^{2}+P Q^{2}+C S^{2}+R S^{2}=D Q^{2}+R Q^{2}+B S^{2}+P S^{2}$

Which is true using lemma-3. 
In the similar manner we can prove $S_{2} S_{4} \perp P R$

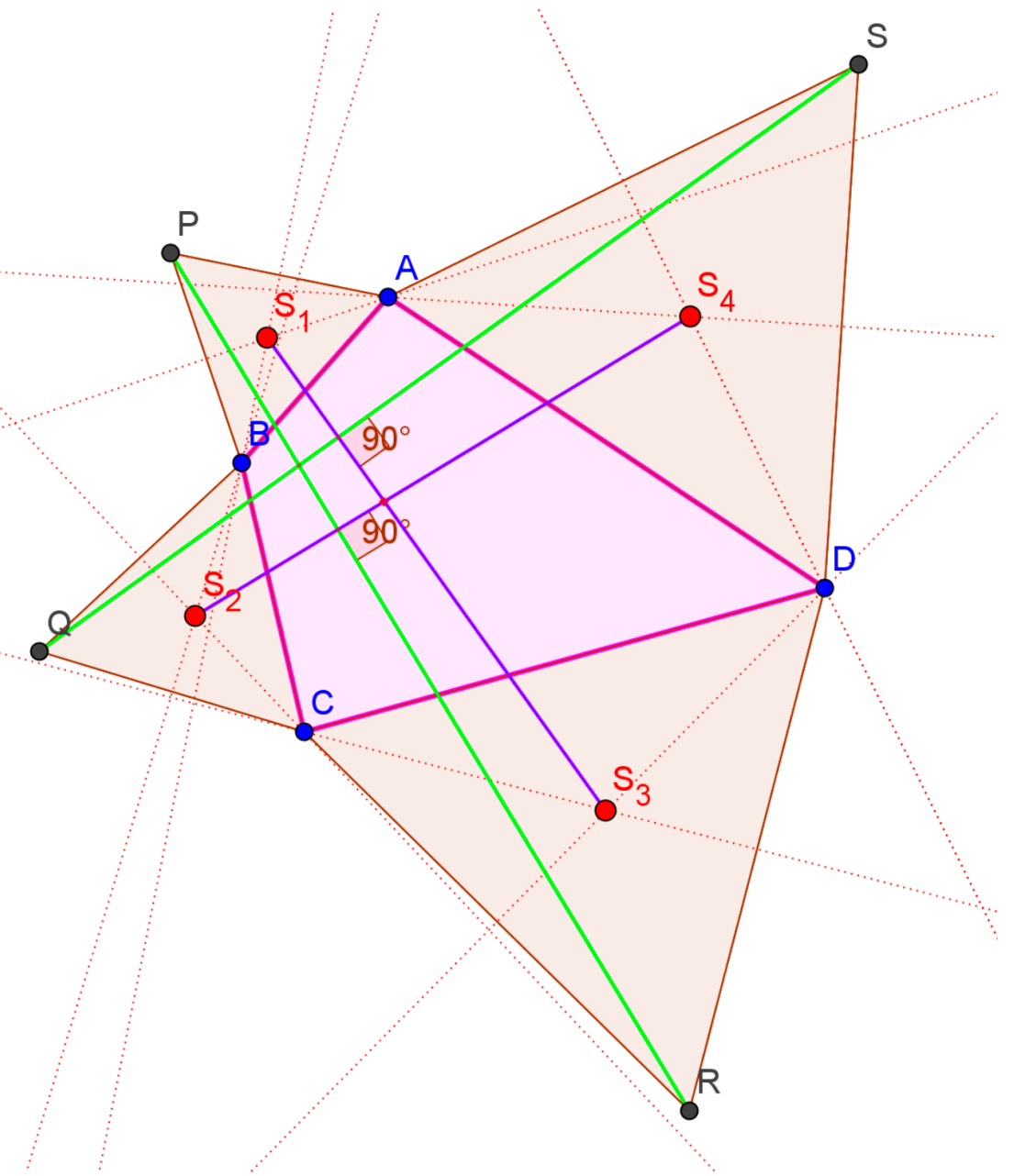

Figure 3

From Figure-3, it is clear $S_{1} S_{3} \perp Q S$ and $S_{2} S_{4} \perp P R$

\section{Theorem-2}

Let $V_{1}, V_{2}, V_{3}$ and $V_{4}$ are the points of intersection of the lines PR, QS, $S_{1} S_{3}$ and $S_{2} S_{4}$ then the four points $V_{1}, V_{2}, V_{3}$ and $V_{4}$ are concyclic (see figure 4).

Proof:

From theorem-1, it is clear that $V_{1} V_{2} \perp V_{2} V_{3}$ and $V_{3} V_{4} \perp V_{4} V_{1}$. Hence the four points $\mathrm{V}_{1}, \mathrm{~V}_{2}, \mathrm{~V}_{3}$ and $\mathrm{V}_{4}$ are concyclic. it completes the proof of the theorem-2. 
6 of 9

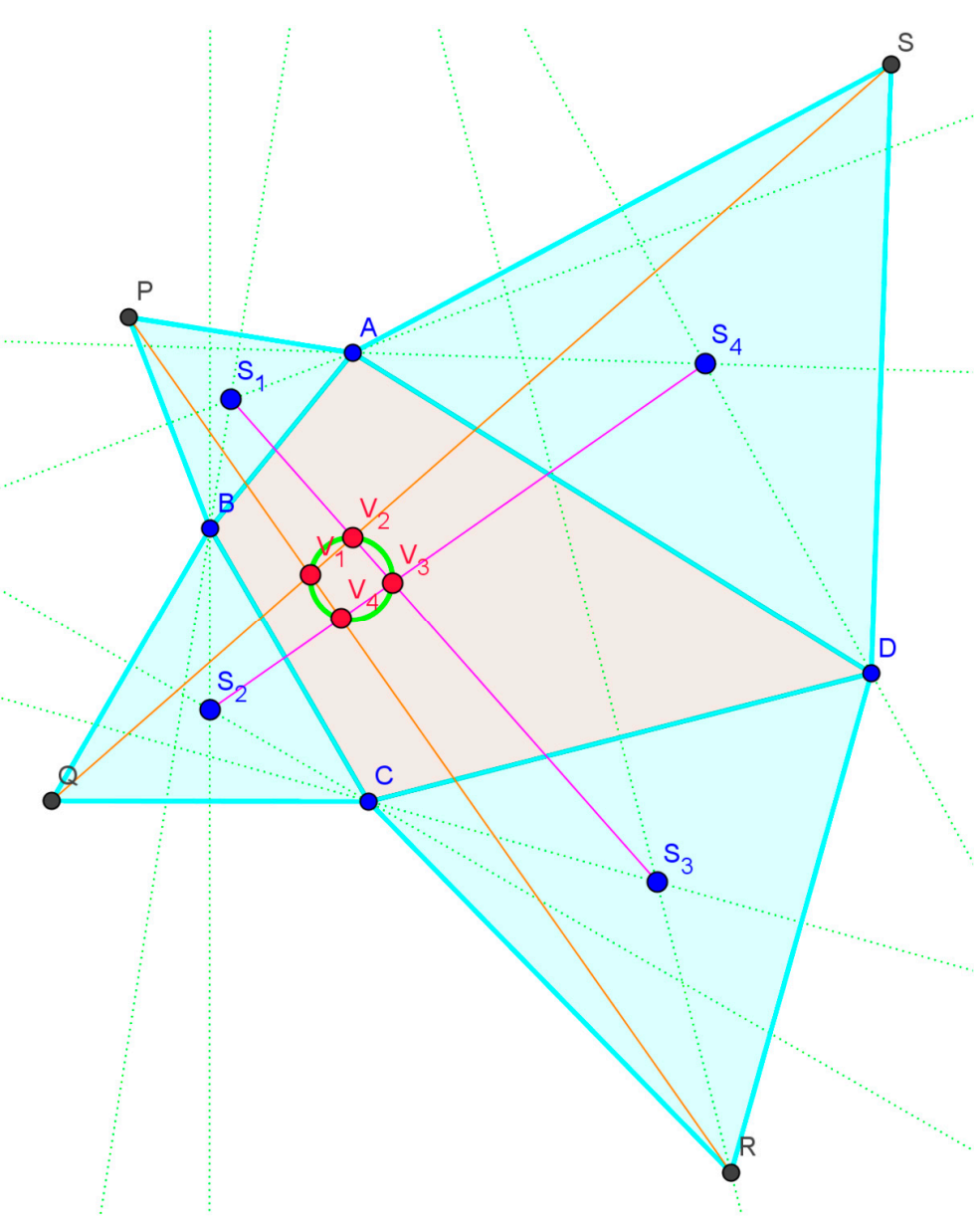

Figure 4

From figure 4, it is clear $\mathrm{V}_{1}, \mathrm{~V}_{2}, \mathrm{~V}_{3} \& \mathrm{~V}_{4}$ are concyclic

\section{Remarks:}

1. Theorem-1 as well as Theorem-2 are also true, even if, the reference quadrilateral is a self- interesecting. (see figure 5 ). 


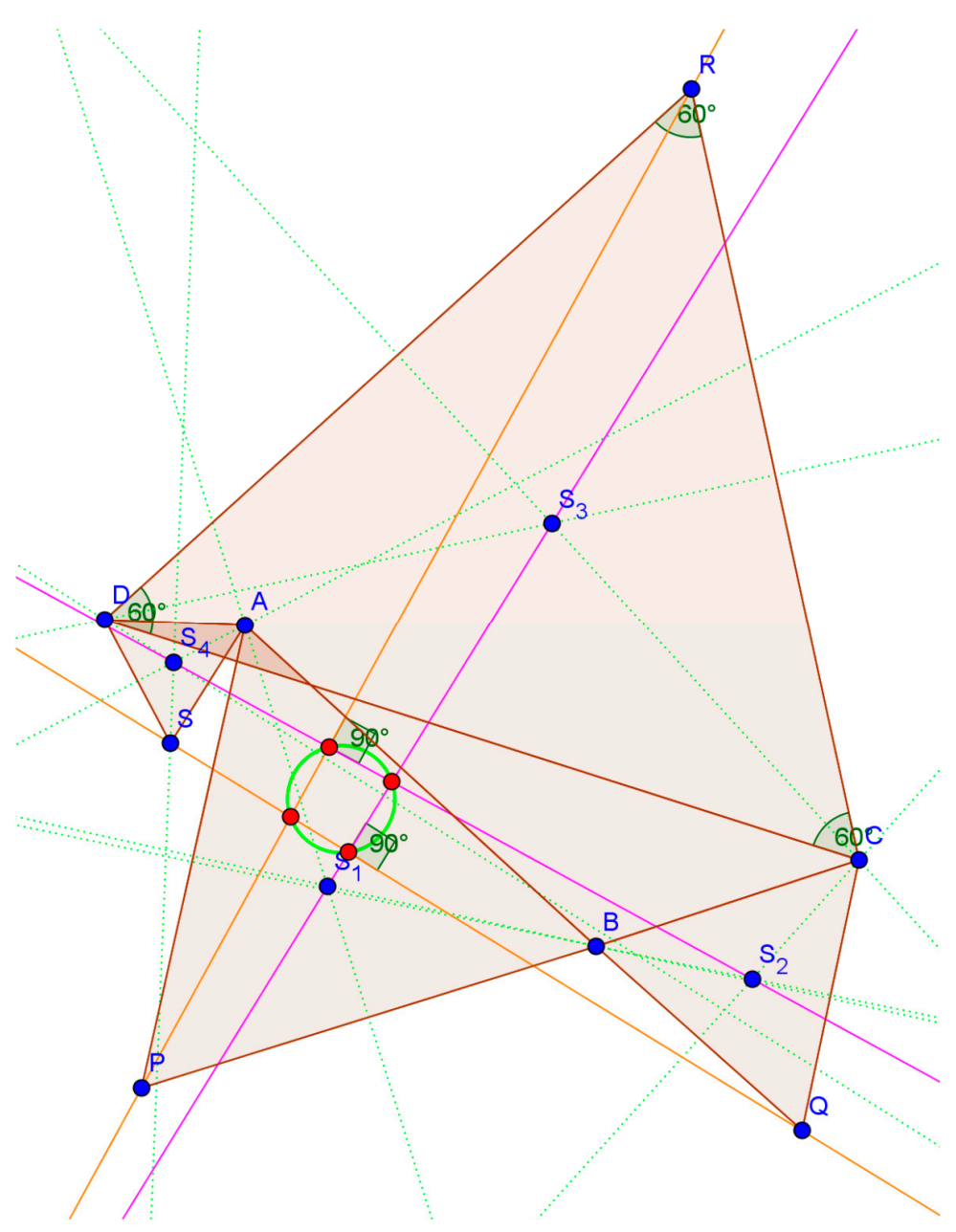

Figure 5

From figure 5, For self intersecting $\mathrm{ABCD}$, it is still true $\mathrm{V}_{1}, \mathrm{~V}_{2}, \mathrm{~V}_{3}, \mathrm{~V}_{4}$ are concyclic and $S_{1} S_{3} \perp Q S \quad S_{2} S_{4} \perp P R$

2. Theorem-1 as well as Theorem-2 are also true, even if, the erected equilateral triangles on the sides of the quadrilateral lie inside. (see figure 6). 
8 of 9

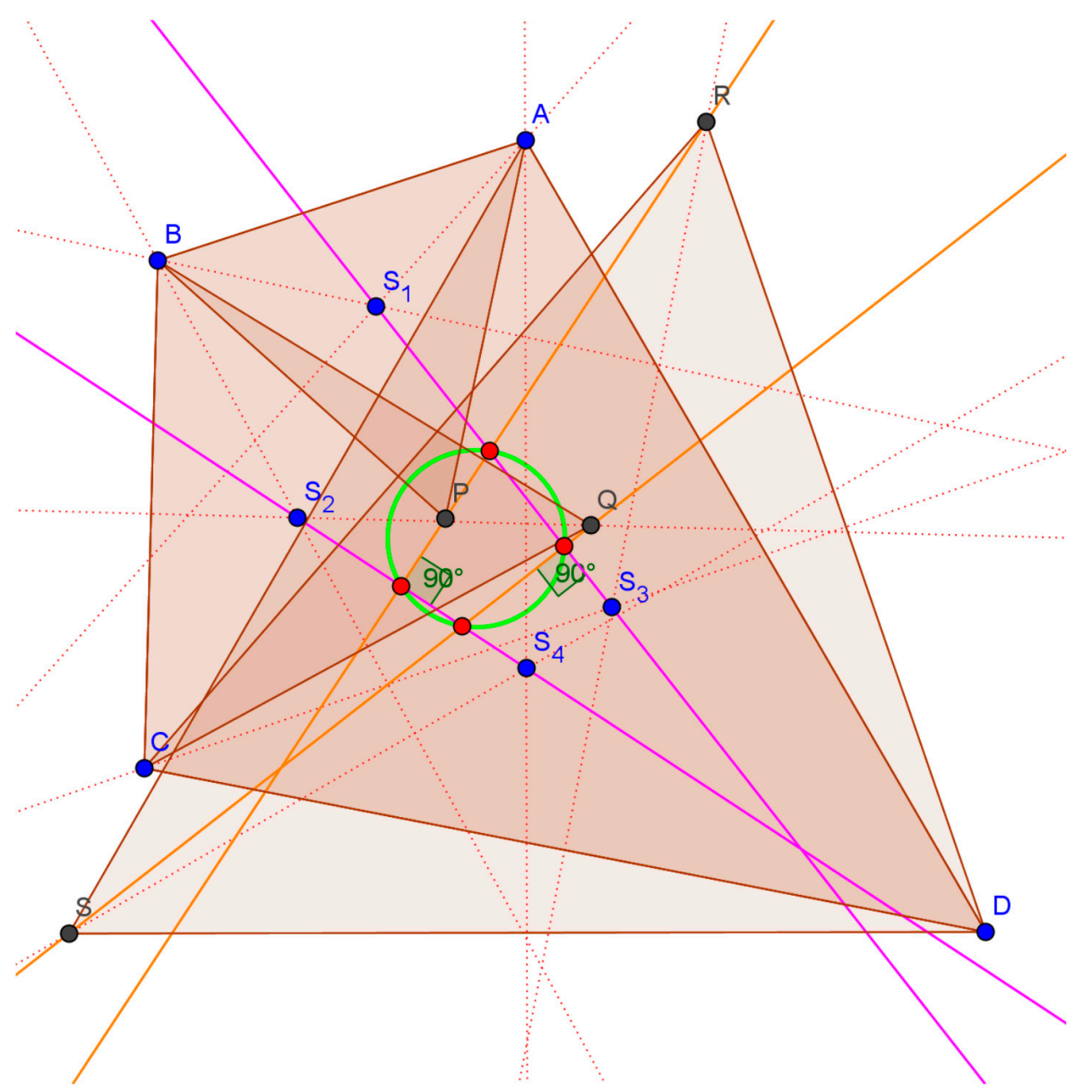

Figure 6

From figure 6, the Equi.Triangles erected inside, it is still true $\mathrm{V}_{1}, \mathrm{~V}_{2}, \mathrm{~V}_{3}, \mathrm{~V}_{4}$ are concyclic and $S_{1} S_{3} \perp Q S \quad S_{2} S_{4} \perp P R$

\section{ACKNOWLEDGEMENT}

The author is would like to thank an anonymous referee for his/her kind comments and suggestions, which lead to a better presentation of this paper. 


\section{References:}

[1]. Dasari Naga Vijay Krishna, Distence Between the Circumcenter and Any Point in the Plane of the Triangle, GeoGebra International Journal of Romania (GGIJRO), art 92, volume5, No.2, 2016.

[2]. Martin Josefsson, Characterizations of Orthodiagonal Quadrilaterals, Forum Geometricorum, Volume 12 (2012) 13-25.

[3]. Michael de Villiers, Generalizing Van Aubel Using Duality, Mathematics Magazine 73 (4), Oct. 2000, pp. 303-307

[4]. Michael de Villiers, Dual generalizations of Van Aubel's theorem, The Mathematical Gazette November (1998), 405-412.

[5]. Yutaka Nishiyama, THE BEAUTIFUL GEOMETRIC THEOREM OF VAN AUBEL, International Journal of Pure and Applied Mathematics Volume 66 No. 1 2011, 71-80.

[6]. http://www.cut-the-knot.org/Curriculum/Geometry/ParaFromTri.shtml.

[7]. http://www.cut-the-knot.org/Curriculum/Geometry/EquiTriOnPara.shtml.

[8]. http://dynamicmathematicslearning.com/vanaubel-application.html.

[9]. http://dynamicmathematicslearning.com/aubelparm.html.

C 2016 by the author; licensee Preprints, Basel, Switzerland. This article is an open access article distributed under the terms and conditions of the Creative Commons by Attribution (CC-BY) license (http://creativecommons.org/licenses/by/4.0/). 